\section{Prehabilitation: Expanding the concept of cardiac rehabilitation}

\author{
Ana Abreu
}

European Journal of Preventive Cardiology

2018, Vol. 25(9) 970-973

(C) The European Society of Cardiology 2018

Reprints and permissions: sagepub.co.uk/journalsPermissions.nav DOI: 10.1 I 177/2047487318763666 journals.sagepub.com/home/ejpc

@SAGE
Cardiac surgery is undoubtedly a major type of acute stress, requiring special clinical attention. Associated morbidity and mortality relate to the underlying disease, surgical technical aspects, patient's general status and many other factors impact on prognosis.

Preoperative cardiac risk assessment needs to determine the presence of poor functional capacity related to increased mortality after thoracic surgery (relative risk $18.7,95 \%$ confidence interval $5.9-59)$. The inability to climb two flights of stairs or run a short distance $(<4$ metabolic equivalents) are associated with postoperative cardiac events. ${ }^{1}$ Also, the evaluation of frailty, defined by physiological resistance decrease and stress vulnerability, ${ }^{2,3}$ must be a preoperative routine, despite being time-consuming. The fast 'eyeball' technique is certainly not enough, and several evaluating methods are available. ${ }^{4}$ Frail patients, usually with medical comorbidities, often present with higher postoperative morbidity and mortality, longer hospital admissions and higher costs. ${ }^{5-8}$ More prevalent in older people, but not exclusive to this age subset, ${ }^{9}$ frailty is associated with a threefold increase in post-surgery mortality and morbidity ${ }^{10}$ relating strongly to major adverse cardiac and cerebrovascular events. ${ }^{11}$

Poor functional capacity and frailty, together with other bad prognostic factors, may be improved postoperatively by cardiac rehabilitation. The clinical value of comprehensive cardiac rehabilitation after coronary or valvular surgery is well defined and recommended, particularly in heart failure, old and debilitated patients. ${ }^{12-15}$ This multidisciplinary intervention, focused on secondary prevention, aims to recover the individual from an acute event, in functional, psychological and social aspects, and to improve quality of life and prognosis. However, a question arises: should the preventive strategy start earlier, involving the preoperative period?

It is quite important to consider that, at present, due to wider acceptance for cardiac surgery, referred patients are frequently old, high risk, disabled and already debilitated. In the next few years, the acceleration in ageing of the population will affect perioperative patient management. It is estimated that the number of patients undergoing surgery in Europe will increase $25 \%$ by 2020 , and for the same period the elderly population will increase over $50 \%$. The total number of surgical procedures will increase even faster because of the rising frequency of interventions with age. ${ }^{16}$ Most probably, preoperative waiting times will also increase. Patients on the waiting list for elective interventions tend to have little physical activity while waiting. ${ }^{17-19}$ It is quite likely, though, to anticipate that they will become worse, in terms of muscle mass, strength, endurance and activity level after surgery. The big problem after cardiac surgery may be that the heart gets better, but the patient may not. Rather than letting the waiting list period be of inactivity and anxiety, this period could be used for improving nutrition, physical exercise, stress management with relaxation techniques and pharmachological optimisation and education, preparing the patient and caregivers for surgery.

Currently, there is limited research about the use of preoperative rehabilitation, so-called 'prehab', in cardiac patients. The studies typically concentrate on cardiopulmonary outcomes and the utilisation of respiratory physical therapy as an intervention, resulting in some reduction in postoperative complications and decreased hospital length of stay. ${ }^{20}$ The use of group exercise in patients awaiting coronary surgery also demonstrated a significant decrease in hospital length of stay, besides the increase in health-related quality of life and improved recruitment to cardiac rehabilitation. ${ }^{21}$ The PREHAB study, ${ }^{22}$ which hypothesised that an interdisciplinary preoperative programme will reduce the proportion of frail, older patients requiring a postoperative hospital length of stay greater than 7 days, is still ongoing and results are still expected this year.

Cardiologist of Cardiology Service, Hospital Santa Marta, Lisbon Cardiology Professor, Medicine Faculty, University of Lisbon, Portugal

\section{Corresponding author:}

Ana Abreu, Rua Nóbrega e Sousa, $\mathrm{N}^{\circ} \mathrm{I}, 14^{\circ} \mathrm{C}$, Alto Lumiar, Lisboa, Portugal

Email: ananabreu@hotmail.com 
A recent systematic review and meta-analysis 'The impact of prehabilitation on post-surgical complications in patients undergoing non-urgent cardiovascular surgical intervention: systematic review and meta-analysis', ${ }^{23}$ by Marmelo et al. has been very important in calling our attention to the possibility and value of improving patients before an invasive intervention, and minimising the risk. It aimed at evaluating the ability of prehabilitation to prevent post-surgical complications in cardiac patients. It reported that intervening in the functional status of a patient before elective surgical interventions would reduce complications and increase maximal inspiratory pressure. It also indicated a non-significant reduction in the length of hospital stay and an improvement in functional capacity, without decreasing mechanical ventilation time.

Conclusions are, however, limited by the small number of patients in each of the studies included in the present meta-analysis. ${ }^{23}$ It does not allow comparisons between different subsets, defined by age, gender, cardiac disease and surgical intervention. With a larger number of patients, several sub-analyses could have been performed. For this reason, questions remain: younger not deconditioned patients will have benefits from prehabilitation? or only deconditioned, old, frail, immobilised or weak patients, with comorbidities and incapacity? Generally speaking, the worst patients are those who usually have a larger margin for recovery... .

Further information, we did not get from the studies in the meta-analysis, is needed: how many patients randomly assigned for the intervention did effectively undergo it? Barriers and adherence to treatment are important and have been extensively studied in cardiac rehabilitation. Empirically, compared with all the barriers to cardiac rehabilitation, it might be easier to perform prehabilitation, because in the first case the patient considers him or herself already treated and cardiac rehabilitation will be considered somewhat accessory, and in the second case he/she, still symptomatic, may consider prehabilitation a necessary preparation for the main treatment. Nevertheless, this issue needs to be addressed.

Another issue that deserves discussion is the application of the term 'prehabilitation' to preoperative exercise or functional training in the studies of the present metaanalysis. ${ }^{23}$ Prehabilitation should refer to the association of the functional component with other components, which were mostly not present. Disagreeing with the authors of the present meta-analysis, who declare that prehabilitation should be based mostly on preoperative physical conditioning, in my opinion the type of prehabilitation programme should be adapted, targeting the 'weak points' of each patient. Despite the importance of functional improvement, other components, such as pharmacological therapy optimisation, cardiovascular risk factor control, nutritional and psychological intervention, still need further study. Prehabilitation, as with rehabilitation, should be as comprehensive as possible, tailored according to the individual risk profile, physical, psychological and social status assessed as part of the perioperative medical history and examination. Also, it should be noted that the clinical condition and concerns of surgical patients often relate to the surgical procedure itself. Approaching and resolving these issues in addition to understanding the underlying clinical conditions should be part of the comprehensive intervention. ${ }^{24}$

The exercise/functional component needs to be directed differently, more to strength or aerobic training, or to inspiratory muscle training or flexibility/coordination. Kinesiotherapy has been recommended, for a long time, for respiratory patients before and after surgery, and inspiratory muscle training will provide a great benefit to heart failure patients due to muscle disease, which is present in this syndrome.

Nutritional intervention on obesity, cachexia, anaemia and sarcopenia is fundamental. Therapy optimisation, with polypharmacy and adherence management, in renal, respiratory and heart failure and in other comorbidities, such as cardiovascular risk factor control, will improve clinically the 'awaiting' and sometimes 'forgotten' patient.

Psychological status dysfunction, like depression or extreme anxiety, can be exacerbated by the surgical anticipation of stress, consequently causing a homeostasis disequilibrium. In the present systematic review, ${ }^{23}$ depression was evaluated only in one study and anxiety in two studies, the last being significantly less frequent in the intervention group, but only in one of the studies. Longer surgery waiting times are associated with a trend towards deterioration in both patients' psychological and physiological condition, suggesting that the overall condition of those awaiting cardiac surgery should be monitored continuously. ${ }^{25}$ It is also important to control anxiety in the family members. It is indicated that as many as $45 \%$ of patients claimed their own health had suffered along with raised levels of anxiety among spouses. ${ }^{26}$

Patients with controlled stress and anxiety, not depressed, well hydrated and nourished, with minor peripheral oedema and lung congestion, no infectious status, with maintained muscle strength and mobility will certainly have better results from surgery.

The ideal duration of prehabilitation is still unknown. The majority of the authors of the studies included in the present meta-analysis ${ }^{23}$ indicated a prehabilitation time of between 2 and 8 weeks before the procedure with sessions' duration of 15-60 minutes. Variation is large and no studies comparing different durations were performed. Of course, the most debilitated patients with less urgent surgeries should 
probably perform longer prehabilitation. Nevertheless, the best duration for prehabilitation, in different subsets of patients, still needs to be defined.

Marmelo et al. ${ }^{23}$ reported fewer complications in the patients doing prehabilitation, included in this metaanalysis; however, we do not know if the samples of group intervention and group control had the same characteristics (left ventricular function, coronary artery disease severity and extension, completeness of revascularisation, length of surgery, comorbidities, frailty, etc.). Safety should be considered in different subsets of patients with consequent programme adjustment. For instance, in aortic stenosis or severe left ventricular dysfunction, safety will depend largely on the protocol used. We cannot extrapolate to a single group of patients the results obtained in a heterogeneous population submitted to different interventions.

It should be noted that there is a bias in the results of this meta-analysis ${ }^{23}$ and of other studies regarding the fact that prehabilitation patients are those who want prehab, who can move and get to the centre. These patients do not represent the reality of many others, old, frail and disabled. Especially for some of the more difficult patients, home-based programmes could be of help. A small exploratory study ${ }^{27}$ has suggested that providing a real-world clinical home-based prehab programme for frail patients undergoing coronary or valve surgery is feasible, and has the potential to improve functional ability and reduce hospital length of stay related to cardiac surgery. The authors of that study think that a frailty score with greater sensitivity may be required to elucidate the influence frailty could have in reducing the length of stay. A large randomised controlled study will be required to reveal the potential beneficial effects of prehab in this patient population.

My final comments are that all interventions, surgical or percutaneous, should be included in a 'clinical package of tailored habilitation' (pre and re), especially for those frail, old, deconditioned, higher risk patients. If these patients go to surgery with a better nutritional, functional and psychological status as a whole, surely they will have a diminished risk of complications. After surgery the cardiac rehabilitation programme will be performed departing from a better general status, so recovery will be easier, faster and probably more complete. For this purpose, a multidisciplinary team needs to be prepared, ideally the same as working in cardiac rehabilitation.

Interesting issues for future research will be the prehab comparative effects according to age, sex, surgery type, the added benefit of prehab to rehabilitation, the best prehab duration, the effects of prehabilitation in optimising pharmacological therapy, nutrition, psychological function and adherence, besides the functional component and the comparison of centre-based and home-based prehab.

The healthcare system, needing to shorten hospital stays, lower the costs and auditing quality of health provision, may provide implementation of the "whole habilitation': prehabilitation and rehabilitation. We just need to prove these benefits, especially in combination, to 'habilitate' our surgical patients completely.

\section{Declaration of conflicting interests}

The author(s) declared no potential conflicts of interest with respect to the research, authorship, and/or publication of this article.

\section{Funding}

The author(s) received no financial support for the research, authorship, and/or publication of this article.

\section{References}

1. Biccard BM. Relationship between the inability to climb two flights of stairs and outcome after major non-cardiac surgery: implications for the pre-operative assessment of functional capacity. Anaesthesia 2005; 60: 588-593.

2. Fried LP, Ferrucci L, Darer J, et al. Untangling the concepts of disability, frailty, and comorbidity: implications for improved targeting and care. J Gerontol A Biol Sci Med Sci 2004; 59: 255-263.

3. Fried LP, Tangen CM, Walston J, et al. Frailty in older adults evidence for a phenotype. J Gerontol A Biol Sci Med Sci 2001; 56: M146-M157.

4. Vigorito C, Abreu A, Ambrosetti M, et al. Frailty and cardiac rehabilitation: a call for action from the EAPC Cardiac Rehabilitation Section. Eur J Prev Cardiol 2017; 24: $577-590$.

5. Green P, Woglom AE, Genereux P, et al. The impact of frailty status on survival after transcatheter aortic valve replacement in older adults with severe aortic stenosis: a single-center experience. JACC Cardiovasc Interv 2012; 5: 974-981.

6. Lee DH, Buth KJ, Martin BJ, et al. Frail patients are at increased risk for mortality and prolonged institutional care after cardiac surgery. Circulation 2010; 121: 973-978.

7. Singh M, Rihal CS, Lennon RJ, et al. Influence of frailty and health status on outcomes in patients with coronary disease undergoing percutaneous revascularization. Circ Cardiovasc Qual Outcomes 2011; 4: 496-502.

8. Sündermann S, Dademasch A, Rastan A, et al. One-year follow-up of patients undergoing elective cardiac surgery assessed with the Comprehensive Assessment of Frailty test and its simplified form. Interact Cardiovasc Thorac Surg 2011; 13: 119-123; discussion 123.

9. Schurmans H, Steverink N, Lindenberg S, et al. Old or frail: what tells us more? J Gerontol A Biol Sci Med Sci 2004; 59: M962-M965.

10. Afilalo J, Eisenberg MJ, Morin JF, et al. Gait speed as an incremental predictor of mortality and major morbidity 
in elderly patients undergoing cardiac surgery. $J$ Am Coll Cardiol 2010; 56: 1668-1676.

11. Sepehri A, Beggs T, Hassan A, et al. The impact of frailty on outcomes after cardiac surgery: a systematic review. J Thorac Cardiovasc Surg 2014; 148: 3110-3117.

12. Eagle KA, Guyton RA, Davidoff R, et al. ACC/AHA Guidelines for Coronary Artery Bypass Graft Surgery: a report of the American College of Cardiology/ American Heart Association Task Force on Practice Guidelines (committee to revise the 1991 Guidelines for Coronary Artery Bypass Graft Surgery). American College of Cardiology/ American Heart Association. J Am Coll Cardiol 1999; 34: 1262-1347.

13. Eagle KA, Guyton RA, Davidoff R, et al. ACC/AHA 2004 Guideline update for coronary artery bypass graft surgery: a report of the American College of Cardiology/ American Heart Association Task Force on Practice Guidelines (committee to update the 1999 Guidelines for Coronary Artery Bypass Graft Surgery). Circulation 2004; 110: e340-e437.

14. Vahanian A, Baumgartner H, Bax J, et al. Guidelines on the management of valvular heart disease: the Task Force on the Management of Valvular Heart Disease of the European Society of Cardiology. Eur Heart J 2007; 28: 230-268.

15. Butchart EG, Gohlke-Barwolf C, Antunes MJ, et al. Recommendations for the management of patients after heart valve surgery. Eur Heart J 2005; 26: 2463-2471.

16. Mangano DT. Perioperative medicine: NHLBI working groups deliberations and recommendations. J Cardiothorac Vasc Anesth 2004; 18: 1-6.

17. Sawatzky JAV, Kehler DS, Ready AE, et al. Prehabilitation program for elective coronary artery bypass graft surgery patients: a pilot randomized controlled study. Clin Rehabil 2014; 28: 648-657.

18. Nery RM and Barbisan JN. Effect of leisure-time physical activity on the prognosis of coronary artery bypass graft surgery. Rev Bras Cir Cardiovasc 2010; 25: 73-78.

19. Mooney M, Fitzsimons D and Richardson G. 'No more couch-potato!' Patients' experiences of a pre-operative programme of cardiac rehabilitation for those awaiting coronary artery bypass surgery. Eur $J$ Cardiovasc Nurs 2007; 6: 77-83.

20. Herdy A, Marcchi P, Vila A, et al. Pre- and postoperative cardiopulmonary rehabilitation in hospitalized patients undergoing coronary artery bypass surgery: a randomized controlled trial. Am J Phys Med Rehabil 2008; 87: 714-719.

21. Arthur HM, Daniels C, McKelvie R, et al. Effect of a preoperative intervention on preoperative and postoperative outcomes in low-risk patients awaiting elective coronary artery bypass graft surgery. A randomized, controlled trial. Ann Intern Med 2000; 133: 253-262.

22. Stammers AN, Kehler DS, Afilalo J, et al. Protocol for the PREHAB study - pre-operative rehabilitation for reduction of hospitalization after coronary bypass and valvular surgery: a randomised controlled trial. BMJ Open 2015; 5: e007250.

23. Marmelo F, Rocha V and Gonçalves D. The impact of prehabilitation on post-surgical complications in patients undergoing non-urgent cardiovascular surgical intervention: systematic review and meta-analysis. Eur J Prev Cardiol 2018; 25: 404-417.

24. Piepoli M, Corra U, Benzer W, et al. Secondary prevention through cardiac rehabilitation: from knowledge to implementation. A position paper from the Cardiac Rehabilitation Section of the European Association of Cardiovascular Prevention and Rehabilitation. Eur $J$ Prev Cardiol 2010; 17: 1-17.

25. McCormick KM, Naimark BJ and Tate RB. Uncertainty, symptom distress, anxiety, and functional status in patients awaiting coronary artery bypass surgery. Heart \& Lung - J Acute Crit Care 2006; 35: 34-45.

26. Mulgan R and Logan RL. The coronary bypass waiting list: a social evaluation. NZ Med J 1990; 103: 371-372.

27. Waite I, Deshpande R, Baghai M, et al. Home-based preoperative rehabilitation (prehab) to improve physical function and reduce hospital length of stay for frail patients undergoing coronary artery bypass graft and valve surgery. J Cardiothor Surg 2017; 12: 91. 